\title{
Searches for flavour violation and flavour changing neutral currents in top quark final states
}

\author{
Nicolas Maximilian Köhler, on behalf of the ATLAS Collaboration ${ }^{a, *}$ \\ ${ }^{a}$ CERN, \\ Espl. des Particules 1, Meyrin, Switzerland \\ E-mail: nicolas.koehler@cern.ch
}

The remarkably large integrated luminosity collected by the ATLAS detector at the highest protonproton collision energy provided by the Large Hadron Collider (LHC) allows to probe the presence of new physics that might break well established symmetries or enhance extremely rare processes in the Standard Model (SM) of particle physics. Two such significant examples are lepton universality and Flavour Changing Neutral Currents (FCNC). Recent measurements involving $B$-meson decays sparked renewed interest in testing lepton universality between tau and light leptons because of observed deviations at the four-standard-deviation level. By selecting events with two opposite sign leptons (muon pairs and electron-muon pairs) and at least two $b$-tagged jets, a highly pure sample of top-quark pair decays is assembled and used to extract a large unbiased sample of $W$ bosons decaying to leptons down to low transverse momenta. A fit to the two dimensional distribution of the transverse momentum and the transverse impact parameter of the lepton is then used to differentiate between leptons originating directly from the $W$ boson and those resulting from the $W$-boson-to-tau-lepton decay chain. This results in a precise measurement of the ratio between the probability for the $W$ boson decay to tau to the probability for its decay to muon.

On the other hand, SM FCNC involving the top-quark decay to another up-type quark and a neutral boson are so small that any measurable branching ratio for such a decay is an indication of new physics. Two searches for FCNC couplings are presented. First, the search for the FCNC coupling between the top quark and the Higgs boson uses events with one electron or muon, missing energy and three or four jets to search for both the production of a single top-quark in association with a Higgs boson or the production of a top-quark pair with one top quark decaying to a Higgs boson and an up or a charm quark. The results are interpreted also in terms of effective field theory couplings describing the $t q H$ vertex. Finally, $81 \mathrm{fb}^{-1}$ of integrated luminosity are used to search for FCNC via the coupling of a top quark, a photon and an up or charm quark in events with one photon, one lepton (electron or muon), one b-tagged jet and missing transverse momentum.

40th International Conference on High Energy physics - ICHEP2020

July 28 - August 6, 2020

Prague, Czech Republic (virtual meeting)

\footnotetext{
${ }^{*}$ Speaker
} 


\section{Introduction}

Over the past decades, the Standard Model (SM) of particle physics has been extensively tested by many experiments which all have proven the model to consistently describe the experimental observations. However, astrophysical observations such as the existence of Dark Matter and Dark Energy as well as the measurement of neutrino oscillations clearly point towards the incompleteness of the SM for describing the interactions of elementary particles. The remarkably large integrated luminosity collected by the ATLAS detector [1] during LHC Run 2 allows to probe the presence of new physics that might break well established symmetries or enhance extremely rare processes in the SM such as lepton flavour universality or flavour changing neutral currents.

\section{Measurement of lepton flavour universality}

A fundamental axiom of the SM is the universality of the couplings of the different generations of leptons to the electroweak gauge bosons, the lepton flavour universality. In particular, it predicts that the branching ratio of $W$ bosons decaying to a charged lepton $\ell \in\{e, \mu, \tau\}$ and a neutrino is equal for all lepton flavours, i.e. $R(\tau / \mu)=\mathcal{B R}\left(W \rightarrow \tau \nu_{\tau}\right) / \mathcal{B} \mathcal{R}\left(W \rightarrow \mu \nu_{\mu}\right)=1$ (when neglecting the phase space effects due to the masses of the decay products on this ratio which are at the level of $\sim 5 \times 10^{-4}$ and hence can be neglected [2]). $R(\tau / \mu)$ has been measured by the four experiments at the Large Electron-Positron Collider (LEP), yielding a combined value of 1.070 0.026 [3] which deviates from the SM expectation of unity by $2.7 \sigma$.

A measurement of this quantity with a novel technique using di-leptonic $t \bar{t}$ events is presented based on $139 \mathrm{fb}^{-1}$ of data recorded with the ATLAS detector in proton-proton ( $p p$ ) collisions at $\sqrt{s}=13 \mathrm{TeV}$ [4]. The measurement relies on the precise knowledge of the branching ratio of $\tau$ leptons decaying to muons by only considering leptonic $\tau$-lepton decays to muons. These have the advantage of smaller reconstruction uncertainties compared to hadronic $\tau$-lepton decays. Muons from leptonic $\tau$ decays are distinguished from prompt muons from the $W$ boson decay using the lifetime of the $\tau$-lepton which affects the absolute muon transverse impact parameter $\left(\left|d_{0}^{\mu}\right|\right)$ and the muon transverse momentum $\left(p_{\mathrm{T}}^{\mu}\right)$ spectra. $\left|d_{0}^{\mu}\right|$ is measured in the $x-y$ plane as the closest distance of approach of the track to the beamline since this way its resolution is independent of the primary vertex resolution. The shape of $\left|d_{0}^{\mu}\right|$ for prompt muons is determined from data in a $Z \rightarrow \mu \mu$ control region.

The measurement exploits top and anti-top pairs decaying into leptons instead of single $W$ boson production since one lepton can be used to trigger the event while no strong kinematic requirement needs to be put on the second lepton, maximising the acceptance of the measurement. Furthermore, the $\left|d_{0}^{\mu}\right|$ and $p_{\mathrm{T}}^{\mu}$ distributions also allow to distinguish between muons from $\tau$-lepton decays and background muons from hadron decays due to their differing shapes (cf. Figure 1).

In this measurement, events passing a single electron $\left(p_{\mathrm{T}}^{e}>27 \mathrm{GeV}\right)$ or muon $\left(p_{\mathrm{T}}^{\mu}>27.3 \mathrm{GeV}\right)$ trigger are selected. Exactly one additional muon of opposite charge and exactly two $b$-tagged jets are required. The leptons are required to be isolated from other activity in the event. In case of the $\mu-\mu$ channel, events with $85 \mathrm{GeV}<m_{\mu \mu}<95 \mathrm{GeV}$ are vetoed to suppress backgrounds arising from $Z$ boson production with additional jets. To suppress Drell-Yan and QCD background, events with $m_{\ell \ell}<15 \mathrm{GeV}$ are vetoed. The muon not used to trigger the event must have a transverse momentum $p_{\mathrm{T}}^{\mu}>5 \mathrm{GeV}$, a distance of approach to the primary vertex in the $r-z$ plane of less than $0.3 \mathrm{~mm}$ and an absolute transverse impact parameter relative to the beamline, $\left|d_{0}^{\mu}\right|$, of less than $0.5 \mathrm{~mm}$. 

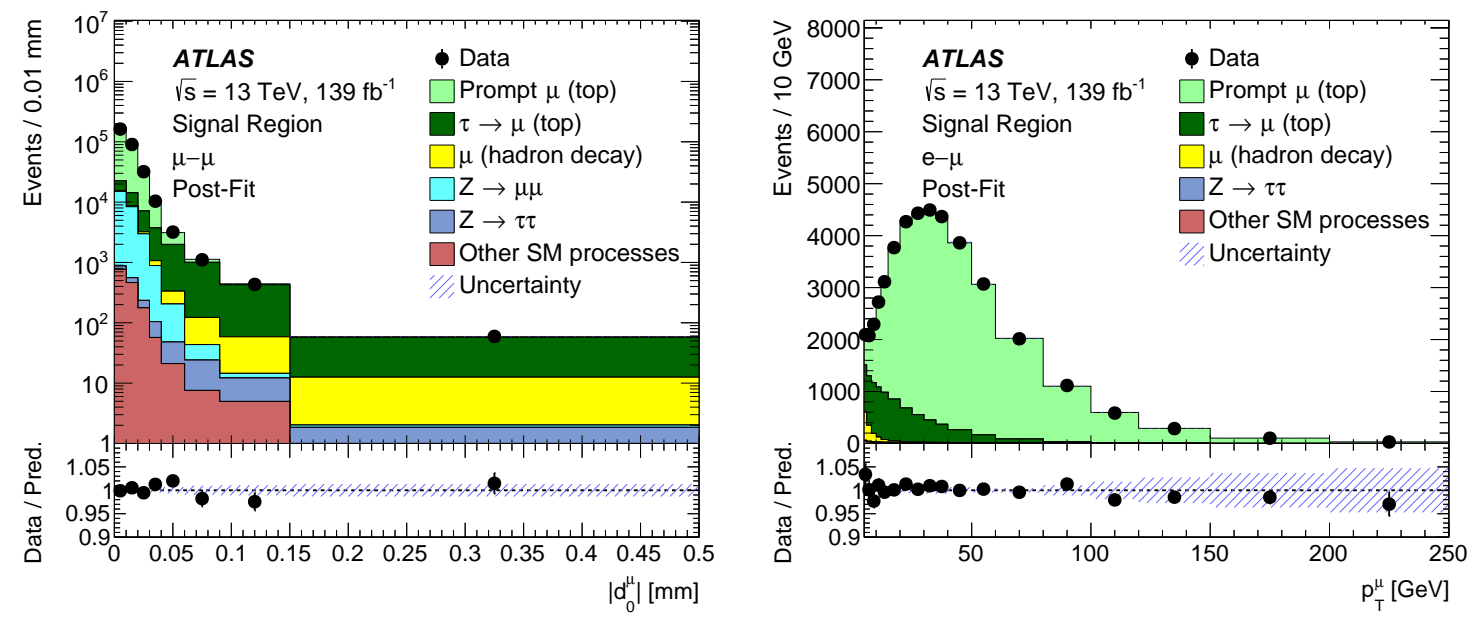

Figure 1: The probe muon $\left|d_{0}^{\mu}\right|$ and $p_{\mathrm{T}}^{\mu}$ distributions for each tag lepton channel (left: $\mu$ - $\mu$ channel, right: $e-\mu$ channel) inclusive in the other variable after the fit to data has been performed. The bottom panel shows the ratio of the data to the expectation. Blue bands indicate the systematic uncertainties with the constraints from the analysis fit applied [4].

After applying this selection, the dominant background contributions arise from $b$ - and $c$ hadrons decaying into muons, and $Z$ boson with additional $b$-tagged jets production in the $\mu-\mu$ channel. All other background processes are fully estimated from Monte Carlo (MC) simulation. The muons from $b$ - and $c$-hadrons mainly contribute at large $\left|d_{0}^{\mu}\right|$ and their contribution is estimated from data in a same sign (SS) control region with the shape of the $\left|d_{0}^{\mu}\right|$ distribution taken from MC. Since at large $p_{\mathrm{T}}^{\mu}$, the SS control region is mainly contaminated by $t \bar{t}+V$ production, the $t \bar{t}+V$ contribution for $p_{\mathrm{T}}^{\mu}>30 \mathrm{GeV}$ taken from MC is subtracted before estimating the normalisation of the background contribution of muons arising from hadron decays. The normalisation of the background arising from $Z$ boson production in association with $b$-tagged jets in the $\mu$ - $\mu$ channel is estimated in a $\mu-\mu$ control region inclusive in the number of jets by fitting the $m_{\mu \mu}$ distribution with a convolution of a Breit-Wigner and a Gaussian for the $Z \rightarrow \mu \mu$ production and a third order Chebychev polonomial for the remaining background processes.

A profile likelihood fit [5] is performed in three bins in $p_{\mathrm{T}}^{\mu}$ and eight bins in $\left|d_{0}^{\mu}\right|$ of the probe muon for each channel $(e-\mu$ and $\mu-\mu)$, making 48 bins in total. Since both $t \bar{t}$ and $W t$ production result in two $W$ bosons being present in the event, in addition to the parameter of interest $R(\tau / \mu)$, a normalisation parameter $k(t \bar{t})$ is added as free parameter to the fit which is taken to be the same for both the $W \rightarrow \mu v_{\mu}$ and $W \rightarrow \tau v_{\tau}$ processes across all bins. Several nuisance parameter values representing the statistical and systematic uncertainties are included when the negative-log-likelihood minimisation is performed. The measured value of $R(\tau / \mu)$ is $R(\tau / \mu)=0.992 \pm 0.013[ \pm 0.007$ (stat) \pm 0.011 (syst) $]$ (cf. Figure 2a), which is consistent with lepton flavour universality and is the most precise measurement to date.

The measurement is dominated by the systematic uncertainties, which are in total 0.011. A breakdown of the systematic uncertainties can be seen in Figure 2b. The dominant systematic uncertainty arises from the data-driven templates for $\left|d_{0}^{\mu}\right|$ accounting for the fact that the templates are estimated in a $Z \rightarrow \mu \mu$ selection but applied to the dileptonic $t \bar{t}$ final state used for the 


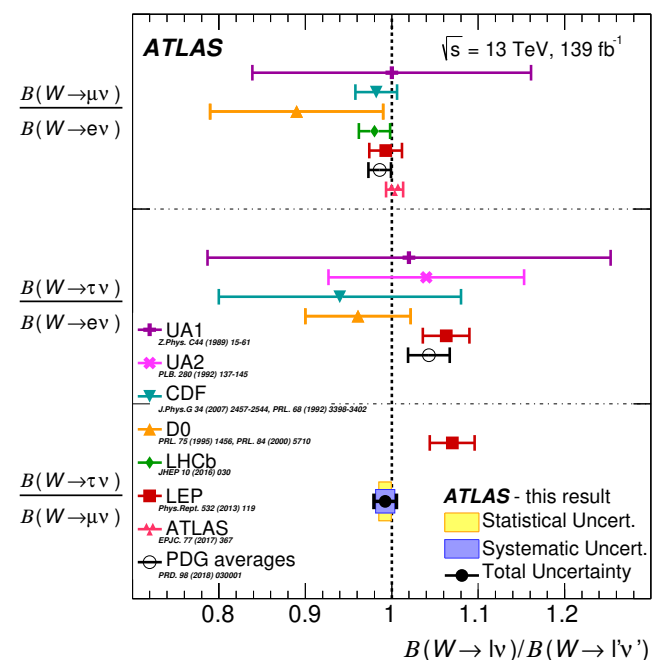

(a) The measured value of $R(\tau / \mu)$ with previous measurements, as well as previous measurements of $R(\mu / e)$ and $R(\tau / e)$. The statistical (yellow box) and systematic (purple box) uncertainties are shown separately as well as the total uncertainty on the measurement (black circular marker). A vertical dashed line indicates the Standard Model prediction of equal branching ratios to different lepton flavours [4].

\begin{tabular}{|l|c|}
\hline Source & Impact on $\boldsymbol{R}(\tau / \boldsymbol{\mu})$ \\
\hline \hline Prompt $d_{0}^{\mu}$ templates & 0.0038 \\
$\mu_{(\text {prompt })}$ and $\mu_{(\tau \rightarrow \mu)}$ parton shower variations & 0.0036 \\
Muon isolation efficiency & 0.0033 \\
Muon identification and reconstruction & 0.0030 \\
$\mu_{(\text {had. })}$ normalisation & 0.0028 \\
$t \bar{t}$ scale and matching variations & 0.0027 \\
Top $p_{\mathrm{T}}$ spectum variation & 0.0026 \\
$\mu_{(\text {had. })}$ parton shower variations & 0.0021 \\
Monte Carlo statistics & 0.0018 \\
Pile-up & 0.0017 \\
$\mu_{(\tau \rightarrow \mu)}$ and $\mu_{(\text {had. }} d_{0}^{\mu}$ shape & 0.0017 \\
Other detector systematic uncertainties & 0.0016 \\
$Z+$ jet normalisation & 0.0009 \\
Other sources & 0.0004 \\
\hline$B\left(\tau \rightarrow \mu v_{\tau} v_{\mu}\right)$ & 0.0023 \\
\hline \hline Total systematic uncertainty & 0.0109 \\
\hline Data statistics & 0.0072 \\
\hline \hline Total & $\mathbf{0 . 0 1 3}$ \\
\hline
\end{tabular}

(b) A list of the sources of uncertainty affecting the measurement. The impact on $R(\tau / \mu)$ is assessed by fixing the relevant fit parameters for a given uncertainty and re-fitting to data. The size of the uncertainty reduction in this modified fit is the quoted impact. Different individual components used in the fit are combined into categories such that the leading sources can be seen [4].

Figure 2

measurement. The second largest uncertainty is due to $t \bar{t}$ modeling uncertainties estimated by comparing different parton shower algorithms in the simulated $t \bar{t}$ and $W t$ datasets. Other dominant systematic uncertainties of the measurement are the efficiencies of the muon reconstruction towards lower $p_{\mathrm{T}}^{\mu}$ as well as the efficiencies of the muon isolation selection. The uncertainties due to the data-driven estimate of the contribution of hadrons decaying to muons are driven by the statistics of the SS control region as well as the choice of MC generator used.

\section{Searches for flavour changing neutral currents}

Although flavour-changing neutral currents (FCNCs) are forbidden at tree level in the SM and strongly suppressed at higher orders via the GIM mechanism [6], there are several extensions of the SM involving FCNCs such as $R$-parity violating SUSY [7], 2HDM [8], composite Higgs [9] or warped extra dimensions models [10]. These models predict a significantly larger probability to observe FCNC processes in LHC collisions.

A search for FCNCs involving a top quark and a photon is performed using $81 \mathrm{fb}^{-1}$ of $p p$ collision data at $\sqrt{s}=13 \mathrm{TeV}$ [11]. The analysis is sensitive to potential FCNC in the production or decay of a top quark. In both cases, the considered primary process consists of one top quark, one photon and additional jets. Only leptonic top quark decays are considered such that events with exactly one isolated lepton that triggered the event, one photon, one $b$-tagged jet and missing transverse energy, $E_{\mathrm{T}}^{\mathrm{miss}}$, are selected. The event selection is sensitive to both left-handed (LH) and 


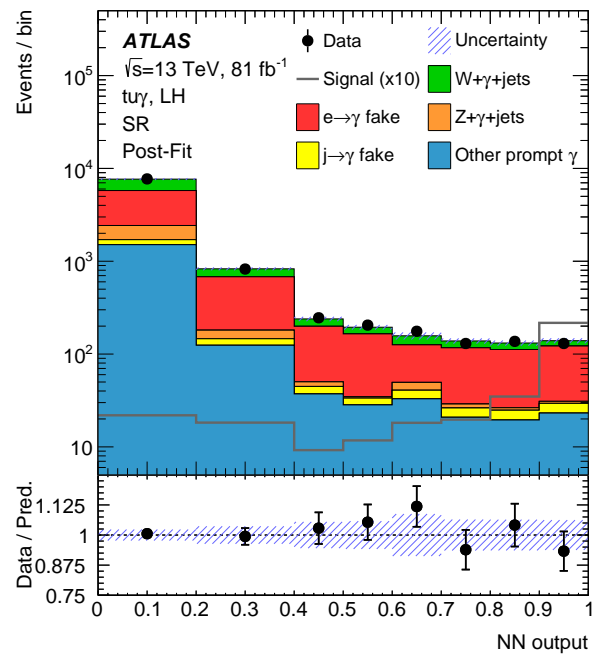

(a) Post-fit distributions of a background-only fit to the SR of the NN output. In addition, the expected signal is overlaid for an effective coupling strength corresponding to the observed limit multiplied by a factor of ten [11].

\begin{tabular}{ccccc}
\hline \multicolumn{1}{c}{ Observable } & Vertex & Coupling & Obs. & Exp. \\
\hline$\left|C_{\mathrm{uW}}^{(13) *}+C_{\mathrm{uB}}^{(13) *}\right|$ & $t u \gamma$ & $\mathrm{LH}$ & 0.19 & $0.22_{-0.03}^{+0.04}$ \\
$\left|C_{\mathrm{uW}}^{(31)}+C_{\mathrm{uB}}^{(31)}\right|$ & $t u \gamma$ & $\mathrm{RH}$ & 0.27 & $0.27_{-0.04}^{+0.05}$ \\
$\left|C_{\mathrm{uW}}^{(23) *}+C_{\mathrm{uB}}^{(23) *}\right|$ & $t c \gamma$ & $\mathrm{LH}$ & 0.52 & $0.57_{-0.09}^{+0.11}$ \\
$\left|C_{\mathrm{uW}}^{(32)}+C_{\mathrm{uB}}^{(32)}\right|$ & $t c \gamma$ & $\mathrm{RH}$ & 0.48 & $0.59_{-0.09}^{+0.12}$ \\
\hline$\sigma(p p \rightarrow t \gamma)[\mathrm{fb}]$ & $t u \gamma$ & $\mathrm{LH}$ & 36 & $52_{-14}^{+21}$ \\
$\sigma(p p \rightarrow t \gamma)[\mathrm{fb}]$ & $t u \gamma$ & $\mathrm{RH}$ & 78 & $75_{-21}^{+31}$ \\
$\sigma(p p \rightarrow t \gamma)[\mathrm{fb}]$ & $t c \gamma$ & $\mathrm{LH}$ & 40 & $49_{-14}^{+20}$ \\
$\sigma(p p \rightarrow t \gamma)[\mathrm{fb}]$ & $t c \gamma$ & $\mathrm{RH}$ & 33 & $52_{-14}^{+22}$ \\
\hline $\mathcal{B}(t \rightarrow q \gamma)\left[10^{-5}\right]$ & $t u \gamma$ & $\mathrm{LH}$ & 2.8 & $4.0_{-1.1}^{+1.6}$ \\
$\mathcal{B}(t \rightarrow q \gamma)\left[10^{-5}\right]$ & $t u \gamma$ & $\mathrm{RH}$ & 6.1 & $5.9_{-1.6}^{+2 .}$ \\
$\mathcal{B}(t \rightarrow q \gamma)\left[10^{-5}\right]$ & $t c \gamma$ & $\mathrm{LH}$ & 22 & $27_{-7}^{+11}$ \\
$\mathcal{B}(t \rightarrow q \gamma)\left[10^{-5}\right]$ & $t c \gamma$ & $\mathrm{RH}$ & 18 & $28_{-8}^{+12}$ \\
\hline
\end{tabular}

(b) Observed (expected) 95\% CL limits on the effective coupling strengths for different vertices and couplings, the production cross section, and the branching ratio. For the former, the energy scale is assumed to be $\Lambda=1 \mathrm{TeV}[11]$.

Figure 3

right-handed (RH) FCNC couplings. A neural network trained on the transverse momenta of the photon, the lepton and the jet, the charge of the lepton, $E_{\mathrm{T}}^{\mathrm{miss}}$, the lepton-photon and lepton-jet invariant masses, and the angular distance between the lepton and the photon, between the lepton and the jet, and between the jet and the photon is used to improve the discrimination between signal and background. The network is trained separately for the FCNC coupling to $u$ - and $c$-quarks and the LH and RH couplings.

The dominant background processes are $W+\gamma+$ jets and $Z+\gamma+$ jets production whose normalisations are estimated from data in dedicated control regions. For $W+\gamma+$ jets, additionally to the signal region selection, a non- $b$-tagged jet and a lepton-photon invariant mass $m_{\ell \gamma}<60 \mathrm{GeV}$ or $m_{\ell \gamma}>100 \mathrm{GeV}$ are required to suppress contributions from $Z+$ jets production. For $Z+\gamma+$ jets, exactly one photon, two oppositely-charged same flavour leptons and no additional requirements on jets or $E_{\mathrm{T}}^{\text {miss }}$ are applied. The number of electrons and hadrons misidentified as photons is estimated from data by defining electron (hadron) fake control regions based on the dielectron or the electron-photon invariant mass measured in $Z$ boson decays (modifying the photon selection criteria).

A binned profile-likelihood fit to the output distribution of the neural network (cf. Fig 3a) is performed taking the statistical and systematic uncertainties into account as nuisance parameters of the fit. The data and SM predictions agree within uncertainties and no significant FCNC contributions are observed. From the $95 \%$ confidence level (CL) limits on the signal contribution, derived using the $C L_{\mathrm{s}}$ method [12], the corresponding limits on the effective coupling parameters are calculated, and from these the limits on the production cross section and branching ratios are calculated (cf. Figure 3b), that are the most stringent to date. 

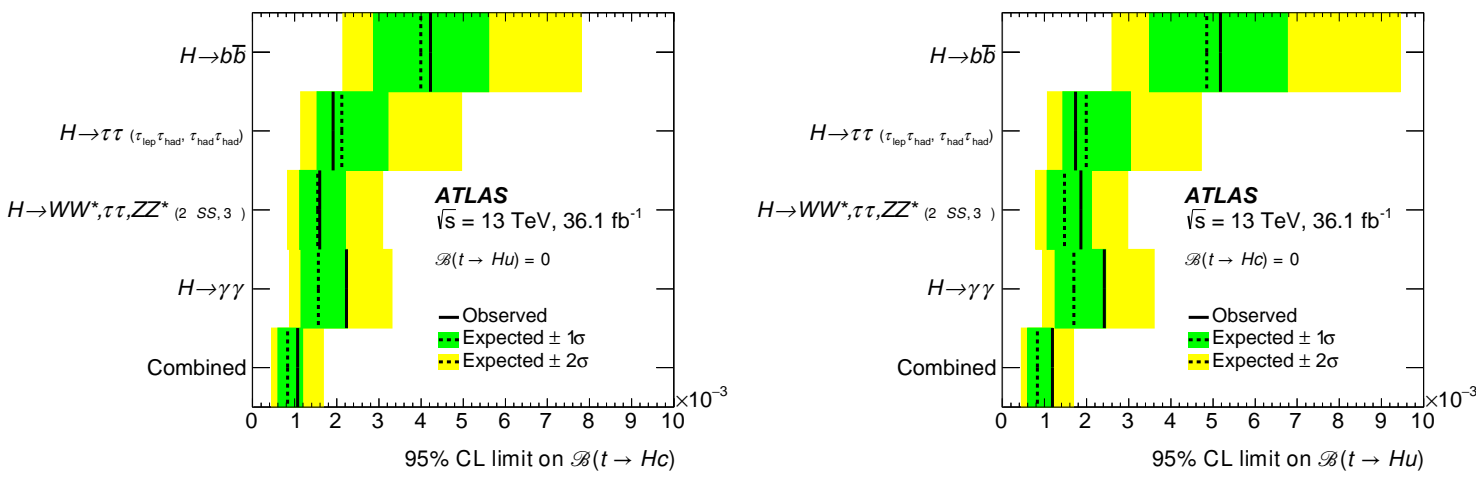

Figure 4: $95 \% \mathrm{CL}$ upper limits on $\mathcal{B}(t \rightarrow H c)(\mathcal{B}(t \rightarrow H u)$ on the right) for the individual searches as well as their combination, assuming $\mathcal{B}(t \rightarrow H u)=0(\mathcal{B}(t \rightarrow H c)=0$ on the right). The observed limits (solid lines) are compared with the expected (median) limits under the background-only hypothesis (dotted lines). The surrounding shaded bands correspond to the $68 \%$ and $95 \%$ CL intervals around the expected limits, denoted by $\pm 1 \sigma$ and $\pm 2 \sigma$, respectively [13].

A search for FCNC decays of a top quark into an up-type quark $(q=u, c)$ and the Standard Model Higgs boson, $t \rightarrow H q$ based on $36.1 \mathrm{fb}^{-1}$ of $p p$ collision data at $\sqrt{s}=13 \mathrm{TeV}$ is performed [13]. Two complementary analyses are performed to search for top-quark pair events in which one top quark decays into $W b$ and the other top quark decays into $H q$, and target the $H \rightarrow b \bar{b}$ and $H \rightarrow \tau^{+} \tau^{-}$ decay modes, respectively. $t q H(b \bar{b})$ events are required to pass a single lepton trigger (electron or muon) and to contain at least four jets, of which at least two must be $b$-tagged. $\operatorname{tqH}\left(\tau^{+} \tau^{-}\right)$events are required to pass a di- $\tau$ trigger in case of $\tau_{\text {had }} \tau_{\text {had }}$ decays and a single lepton trigger in case of $\tau_{\text {lep }} \tau_{\text {had }}$ decays. In addition, at least three jets and exactly one $b$-tagged jet are required.

The main background process after applying this preselection is $t \bar{t}$ production in association with jets. All backgrounds involving prompt leptons are fully estimated from MC whereas all backgrounds with non-prompt leptons or photons and jets misidentified as electrons or hadronic $\tau$-decays are estimated using data-driven techniques.

The sensitivity of the $t q H(b \bar{b})$ final state is increased by categorising the events into the number of jets $(4,5$ and $\geq 6)$ and on the number of $b$-tagged jets $(2,3$ and $\geq 4)$ and defining a likelihood discriminant based on the four-momenta of the lepton and the jets (including the $b$ tagging information) and the $E_{\mathrm{T}}^{\text {miss }}$. For the $t q H\left(\tau^{+} \tau^{-}\right)$final state, the selected events are categorised into four signal regions depending on the number of $\tau_{\text {lep }}$ and $\tau_{\text {had }}$ candidates and on the number of jets. A boosted decision tree trained on the invariant masses of the decay products, the transverse momentum of the lepton and the momentum fractions carried by the visible decay products of the $\tau$-leptons is used as the discriminating variable in the binned likelihood fit.

In both final states, no significant excess above the SM expectation is observed and limits and 95\% CL upper limits on the $t \rightarrow H q$ branching ratios are derived (cf. Figure 4). The combination of these searches with ATLAS searches in diphoton and multilepton final states yields observed 95\% CL upper limits on the $t \rightarrow H c$ and $t \rightarrow H u$ branching ratios of $1.1 \cdot 10^{-3}$ and $1.2 \cdot 10^{-3}$, assuming $\mathcal{B}(t \rightarrow H u)=0$ and $\mathcal{B}(t \rightarrow H c)=0$, respectively [13]. 


\section{Conclusion}

A measurement of $R(\tau / \mu)$ has been carried out by the ATLAS experiment using $139 \mathrm{fb}^{-1}$ of $p p$ collision data recorded at $\sqrt{s}=13 \mathrm{TeV}$ at the LHC. The result agrees well with the Standard Model prediction and rules out the discrepancy previously observed by the LEP experiments.

Furthermore, two searches for flavour changing neutral currents in top quark final states based on $81 \mathrm{fb}^{-1}$ and $36.1 \mathrm{fb}^{-1}$ of $p p$ collision data were performed. In those searches, a sophisticated background suppression is crucial to gain the required sensitivity. This is achieved using different machine learning techniques. No sign for flavour changing neutral currents involving top quarks is observed.

\section{References}

[1] ATLAS Collaboration: The ATLAS Experiment at the CERN Large Hadron Collider. JINST 3 (2008) S08003.

[2] Particle Data Group, Review of Particle Physics, Phys. Rev. D 98 (2018) 030001.

[3] LEP Electroweak Working Group: Electroweak measurements in electron-positron collisions at $W$-boson-pair energies at LEP. Phys. Rept. 532 (2013) 119.

[4] ATLAS Collaboration: Test of the universality of $\tau$ and $\mu$ lepton couplings in $W$-boson decays from $t \bar{t}$ events with the ATLAS detector. arXiv:2007.14040, 2020.

[5] G. Cowan et al.: Asymptotic formulae for likelihood-based tests of new physics. Eur. Phys. J. C 71 (2011) 1554, Erratum: Eur. Phys. J. C 73 (2013) 250.

[6] S. L. Glashow, J. Iliopoulos and L. Maiani: Weak Interactions with Lepton-Hadron Symmetry. Phys.Rev. D 2 (1970) 1285.

[7] J. M. Yang, B.-L. Young and X. Zhang: Flavor-changing top quark decays in $R$-parity-violating supersymmetric models. Phys. Rev. D 58 (1998) 055001.

[8] D. Atwood, L. Reina and A. Soni: Phenomenology of two Higgs doublet models with flavorchanging neutral currents. Phys. Rev. D 55 (1997) 3156.

[9] A. Azatov et al.: On the flavor structure of natural composite Higgs models \& top flavor violation. JHEP 12 (2014) 082.

[10] A. Azatov, M. Toharia and L. Zhu: Higgs mediated flavor changing neutral currents in warped extra dimensions. Phys. Rev. D 80 (2009) 035016.

[11] ATLAS Collaboration: Search for flavour-changing neutral currents in processes with one top quark and a photon using $81 \mathrm{fb}^{-1}$ of $p p$ collisions at $\sqrt{s}=13 \mathrm{TeV}$ with the ATLAS experiment. Phys. Lett. B 800 (2020).

[12] A. L. Read: Presentation of search results: the $C L_{\mathrm{s}}$ technique. J. Phys. G 28 (2002) 2693.

[13] ATLAS Collaboration: Search for top-quark decays $t \rightarrow H q$ with $36 \mathrm{fb}^{-1}$ of $p p$ collision data at $\sqrt{s}=13 \mathrm{TeV}$ with the ATLAS detector. JHEP 05 (2019) 123.

[14] ATLAS Collaboration: Top Quark FCNC Summary Plots. ATL-PHYS-PUB-2019-038, 2019, https://cds.cern.ch/record/2691201. 